\title{
Diagnosing metabolic acidosis in chronic kidney disease: importance of blood pH and serum anion gap
}

\author{
Jun-Ya Kaimori ${ }^{1,2}$, Yusuke Sakaguchi ${ }^{1,2}$, Sachio Kajimoto ${ }^{2}$, Yuta Asahina ${ }^{2}$, Tatsufumi Oka ${ }^{2}$, Koki Hattori ${ }^{2}$, \\ Yohei Doi ${ }^{2}$, Yoshitaka Isaka ${ }^{2}$ \\ ${ }^{1}$ Department of Inter-Organ Communication Research in Kidney Diseases, Osaka University Graduate School of Medicine, Osaka, Japan \\ ${ }^{2}$ Department of Nephrology, Osaka University Graduate School of Medicine, Osaka, Japan
}

\begin{abstract}
Metabolic acidosis is one of the most common complications of chronic kidney disease (CKD). It is associated with the progression of CKD, and many other functional impairments. Until recently, only serum bicarbonate levels have been used to evaluate acid-base changes in patients with reduced kidney function. However, recent emerging evidence suggests that nephrologists should reevaluate the clinical approach for diagnosing metabolic acidosis in patients with CKD based on two perspectives; pH and anion gap. Biochemistry and physiology textbooks clearly indicate that blood $\mathrm{pH}$ is the most important acid-base parameter for cellular function. Therefore, it is important to determine if the prognostic impact of hypobicarbonatemia varies according to $\mathrm{pH}$ level. A recent cohort study of CKD patients showed that venous $\mathrm{pH}$ modified the association between a low bicarbonate level and the progression of CKD. Furthermore, acidosis with a high anion gap has recently been recognized as an important prognostic factor, because veverimer, a nonabsorbable hydrochloride-binding polymer, has been shown to improve kidney function and decrease the anion gap. Acidosis with high anion gap frequently develops in later stages of CKD. Therefore, the anion gap is a time-varying factor and renal function (estimated glomerular filtration rate) is a time-dependent confounder for the anion gap and renal outcomes. Recent analyses using marginal structural models showed that acidosis with a high anion gap was associated with a high risk of CKD. Based on these observations, reconsideration of the clinical approach to diagnosing and treating metabolic acidosis in CKD may be warranted.
\end{abstract}

Keywords: Anion gap, Hydrogen-ion concentration, Metabolic acidosis

\section{Introduction}

Metabolic acidosis is one of the most common complications in patients with chronic kidney disease (CKD) [1]. This condition should not be overlooked by nephrologists in clinical settings because it has been associated with a wide range of poor outcomes including bone demineralization
[2], insulin resistance [3], muscle protein proteolysis [4], functional limitations in older individuals [5], and cognitive impairment [6]. Importantly, metabolic acidosis is also associated with cardiovascular outcomes and mortality in CKD patients [7-9]. Basic studies showed that acid retention induced by nephron loss or dietary acid load causes kidney tissue injury through endothelin-1 activation, the

Received: September 7, 2021; Revised: October 7, 2021; Accepted: October 18, 2021

Correspondence: Jun-Ya Kaimori

Department of Inter-Organ Communication Research in Kidney Diseases and Department of Nephrology, Osaka University Graduate School of Medicine, 2-2 Yamadaoka, Suita, Osaka 565-0871, Japan. E-mail: kaimori@kid.med.osaka-u.ac.jp

ORCID: https://orcid.org/0000-0001-6892-3942

Copyright (C) 2022 by The Korean Society of Nephrology

() This is an Open Access article distributed under the terms of the Creative Commons Attribution Non-Commercial and No Derivatives License (http:// creativecommons.org/licenses/by-nc-nd/4.0/) which permits unrestricted non-commercial use, distribution of the material without any modifications, and reproduction in any medium, provided the original works properly cited. 
renin-angiotensin-aldosterone system, and the alternative complement pathway [10-13]. In contrast, in several clinical cohort studies, low serum bicarbonate levels were shown to be associated with a faster progression of CKD [14-18]. Indeed, randomized controlled trials and corresponding meta-analyses revealed that alkali therapy conferred beneficial effects against the progression of CKD to kidney failure with replacement therapy (KFRT) [19-24].

According to current guidelines, alkali therapy initiation is recommended when serum bicarbonate levels are $<22$ $\mathrm{mEq} / \mathrm{L}[25,26]$. However, this recommendation is based exclusively on serum bicarbonate levels (Fig. 1). Additionally, clinical trials of veverimer, a recent novel approach for treating metabolic acidosis, have highlighted the possibility that anion gap acidosis is an important cause of CKD progression. In this review, our objective was to reconsider the effects of metabolic acidosis on the progression of CKD from two different perspectives: blood $\mathrm{pH}$ and the anion gap.

\section{Blood pH modulates the association between low bicarbonate level and progression of chronic kidney disease to kidney failure with replacement therapy}

Normal $\mathrm{H}^{+}$concentration in extracellular fluid is almost one-millionth of the concentrations of $\mathrm{N}^{+}, \mathrm{K}^{+}, \mathrm{Cl}^{-}$, and $\mathrm{HCO}_{3}-$. However, compared with larger cations, such as $\mathrm{Na}^{+}$ or $\mathrm{K}^{+}$, small $\mathrm{H}^{+}$ions have stronger affinities for small and negatively charged parts of molecules. Therefore, smaller fluctuations in $\mathrm{H}^{+}$concentrations are required for normal cellular functions [27].
A textbook on acid-base physiology outlines that an initial diagnosis of acid-base disorders should begin with measuring the blood $\mathrm{pH}$ [28]. However, blood $\mathrm{pH}$ is not frequently measured in certain clinical settings, such as in the United States. Rather, the serum total $\mathrm{CO}_{2}\left(\mathrm{TCO}_{2}\right)$ is measured to screen for acid-base disturbances, because $\mathrm{TCO}_{2}$ is affected by both metabolic and respiratory disorders. The screening test ranges from US\$26-\$33 per test. In contrast, in Japan, a venous blood gas test, that includes measuring $\mathrm{Na}^{+}, \mathrm{K}^{+}, \mathrm{Cl}^{-}$, $\mathrm{pH}, \mathrm{pO}_{2}, \mathrm{pCO}_{2}$, and $\mathrm{HCO}_{3}-$, is routinely performed to diagnose acid-base disorders in the clinical outpatient setting [29]. This venous blood gas test costs US $\$ 126$ based on current exchange rates (US $\$ 1$ is almost 110 Japanese yen). Importantly, this cost was established by the Japanese government and all Japanese patients are required to have medical insurance; therefore, Japanese clinicians may not be aware of the direct cost as this is covered by patient insurance.

In the United States, serum $\mathrm{TCO}_{2}$ is used as a surrogate marker of $\mathrm{HCO}_{3}-$. In Japan, $\mathrm{pH}$ and $\mathrm{pCO}_{2}$ in venous blood are measured in clinical laboratories and blood $\mathrm{HCO}_{3}-$ is calculated using the Henderson-Hasselbalch equation [30]. In the United States, blood gas measurements are rarely performed in the main clinical laboratory and typically assessed in a blood gas laboratory [30], which may not be located in a convenient location for patient referrals from clinics. Further, to minimize errors, the measurements should be taken soon after the blood gas samples are obtained. These barriers to measuring blood gases may account for why blood pH is not frequently measured in the outpatient settings.

As described above, acid-base disorder diagnoses and

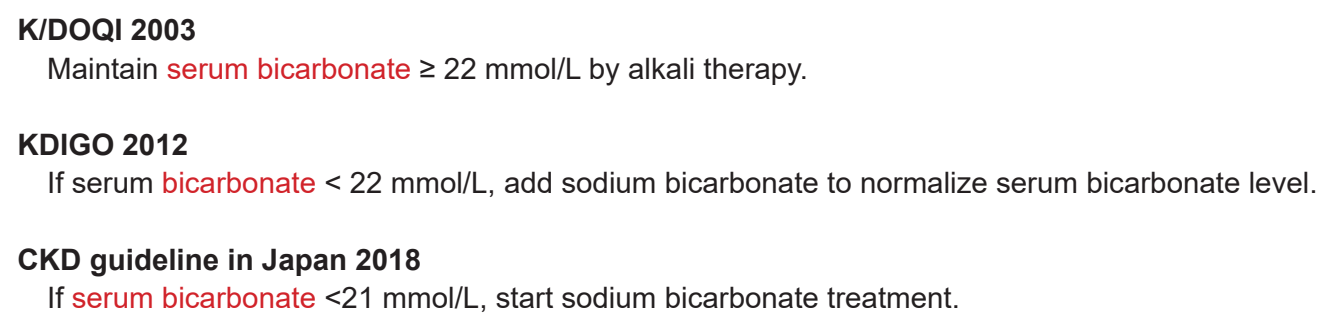

Figure 1. Recommendations for metabolic acidosis management in CKD patients.

CKD, chronic kidney disease; KDIGO, Kidney Disease: Improving Global Outcomes; K/DOQI, Kidney Disease Outcomes Quality Initiative. 
prescriptions for alkali therapy were based exclusively on blood $\mathrm{HCO}_{3}-$ test values. However, the degree to which alterations in blood $\mathrm{pH}$ affect $\mathrm{CKD}$ progression remains unclear. Recently, Kajimoto et al. [31] addressed this important issue by conducting a retrospective cohort study of Japanese CKD patients. In this approach, they used $\mathrm{pH}$ data that were measured along with other parameters in blood gas analyses and analyzed hazard ratios for incident KFRT using Cox proportional hazard models with/without acidemia $(\mathrm{pH}$ $\$ 7.32$ ). Kajimoto et al. [31] evaluated pulmonary diseases, such as chronic obstructive pulmonary disease or interstitial pneumonia in CKD patients and estimated respiratory compensation capacity using venous blood gas data (Fig. 2). They calculated respiratory compensation capacity using large amounts of blood gas data. We plotted the pressure of carbon dioxide against bicarbonate levels and calculated the slope of the regression line using a mixed-effect model. In this context, the slope of the regression line represents the respiratory compensation capacity and represents how much the carbon dioxide pressure can be reduced for each 1-mmol/L decrease in bicarbonate (Fig. 3).

The cohort included 1,058 CKD patients, among which a total of 374 developed KFRT during the median follow-up of 3.0 years. This study determined that $38 \%$ of CKD patients with hypobicarbonatemia $\left(\mathrm{HCO}_{3}-\leq 21.5\right)$ had normal $\mathrm{pH}(7.32 \leq \mathrm{pH} \leq 7.42)$, whereas $59 \%$ with the same $\mathrm{HCO}_{3}-$ values had acidemia $(\mathrm{pH}<7.32)$. These data indicated that approximately $40 \%$ of CKD patients with hypobicarbonatemia $\left(\mathrm{HCO}_{3}-\leq 21.5\right)$ did not have acidemia, which resulted from an adequate respiratory compensation capacity. This implies that a substantial proportion of patients within the

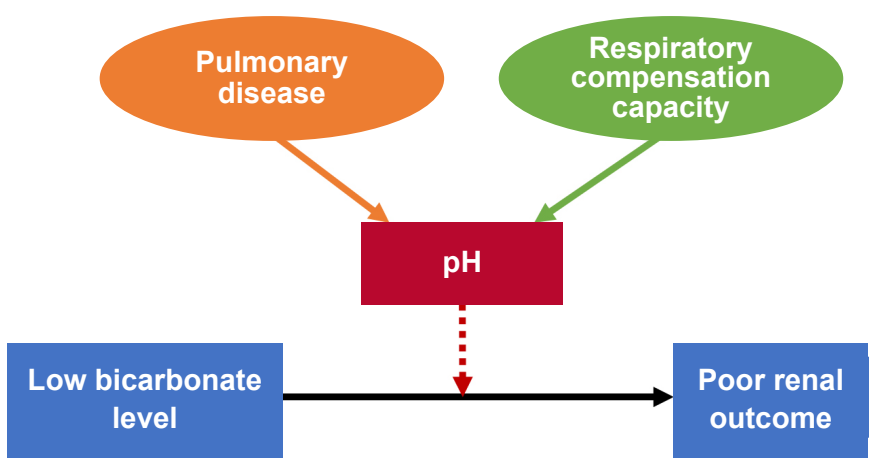

Figure 2. A schematic summary of the study conducted by Kajimoto et al. [31].

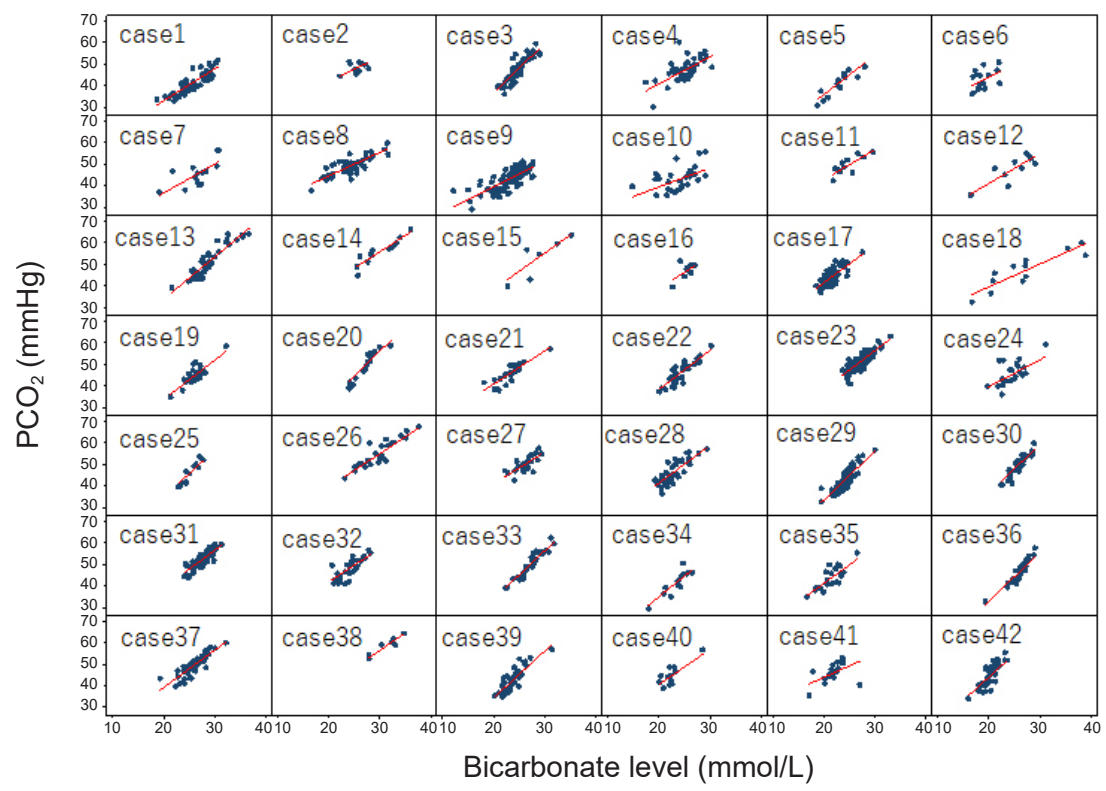

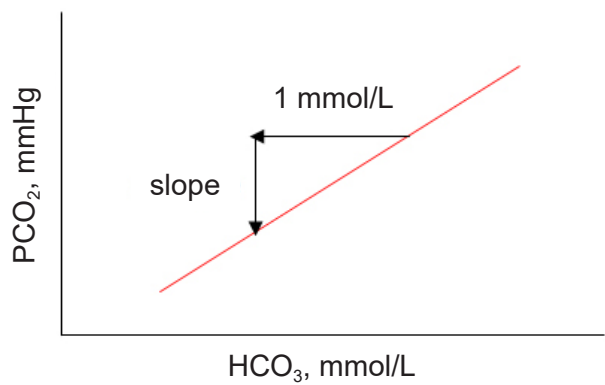

Respiratory compensation capacity = slope of regression line

= how much $\mathrm{PCO}_{2}$ can be reduced if $1 \mathrm{mmol} / \mathrm{L}$ of $\mathrm{HCO}_{3}$ is reduced.

Figure 3. Quantification of respiratory compensation capacity in each CKD patient in the study by Kajimoto et al [31]. The authors assessed the respiratory compensation capacity by using a large number of blood gas data samples. They plotted the pressure of carbon dioxide and bicarbonate levels and calculated the slope of regression line by using a mixed effect model. Here, respiratory compensation capacity is the slope of the regression line, which also reflects the amount of pressure of carbon dioxide that can be reduced if $1 \mathrm{mmol} / \mathrm{L}$ of bicarbonate is reduced.

CKD, chronic kidney disease. 
indicated target range for alkali therapy do not exhibit acidemia. The same observation was made in healthy individuals described in the Health ABC study. Approximately $60 \%$ of individuals with low bicarbonate levels were determined not to have acidemia [32]. Among CKD patients with acidemia ( $\mathrm{pH}<7.32$ ), the lowest bicarbonate quartile exhibited a 2.29-fold higher risk of KFRT compared with the highest bicarbonate quartile. In contrast, among patients without acidemia ( $\mathrm{pH} \geq 7.32$ ), the risk of KFRT in the lowest bicarbonate quartile was not significantly different from that in the highest bicarbonate quartile. In summary, a substantial proportion of CKD patients with hypobicarbonatemia may not be at risk for KFRT, but these patients should be considered as targets for alkali therapy.

With respect to the possibility of increased blood pressure and sodium retention induced by alkali therapy, previous physiologic studies have suggested that $\mathrm{NaHCO}_{3}$ is easier to excrete than $\mathrm{NaCl}$ because $\mathrm{HCO}_{3}$ - is excreted predominantly as $\mathrm{NaHCO}_{3}$, and not as $\mathrm{KHCO}_{3}$ [33]. Therefore, when dietary sodium intake was restricted to approximately 200$700 \mathrm{mg} /$ day, alkali therapy (200 mEq/day, $16.8 \mathrm{~g} /$ day NaH$\mathrm{CO}_{3}$ ) did not induce increases in blood pressure or body weight in a small number of CKD patients [33]. However, a comparable intake of $\mathrm{NaHCO}_{3}(100 \mathrm{mEq} /$ day, $8.4 \mathrm{~g} /$ day $)$ and $\mathrm{NaCl}$ (100 mEq/day, $5.85 \mathrm{~g} /$ day) still induced increases in blood pressure and weight gain [34]. In the general clinical setting, CKD patients do not typically adhere to recommendations to follow very strict restrictions for dietary sodium intake. A recent analysis of CKD patients found that the median salt intake was $8 \mathrm{~g} /$ day [35]. Indeed, recent alkali therapy trials excluded patients with uncontrolled hypertension and/or obvious congestive heart failure [36] and patients with decompensated heart failure [22]. Accordingly, CKD patients that meet criteria for alkali therapy should be selected carefully. The report by Kajimoto et al. [31] may provide important guidance for selecting the most appropriate CKD patients for alkali therapy. According to the study, CKD patients with low bicarbonate levels without acidemia may not require sodium bicarbonate. However, subclinical metabolic acidosis with normal serum bicarbonate has recently emerged and is suggested to have clinical significance [37]. In addition, a previous study revealed that alkali therapy caused greater renal function preservation in patients with normal venous total $\mathrm{CO}_{2}$ [20]. Therefore, additional clinical evidence is needed to address the question of which patients will benefit the most from alkali therapy.

\section{Anion gap levels impact the progression of chronic kidney disease to kidney failure with replacement therapy}

Two categories of metabolic acidosis have been defined based on anion gap levels; normal anion gap (hyperchloremic) acidosis and high anion gap acidosis. Normal anion gap acidosis is usually identified during the early course of CKD, whereas high anion gap acidosis occurs in later stages of CKD owing to the accumulation of nonchloride anions, including phosphate, sulfate, and a wide range of organic acids [38]. As previously described, low bicarbonate levels have been associated with rapid progression of CKD [14-18]. However, the current clinical understanding of how high anion gap acidosis affects renal outcomes, notably in the later stages of CKD is limited. Some initial studies have reported that uremic acids such as indoxyl sulfate, p-cresyl sulfate, and trimethylamine $\mathrm{N}$-oxide cause renal fibrosis that is induced by kidney injury [39-42].

A recent series of clinical trials of veverimer, a nonabsorbable binding polymer for hydrochloric acid, showed intriguing results that are relevant for therapeutic strategies for metabolic acidosis in CKD [43-45]. Veverimer (TRC101) was developed as a treatment for metabolic acidosis in CKD patients. Veverimer is an orally administered, sodium- and counterion-free hydrochloric acid binder and hydrochloric acid binding is a novel therapeutic concept for treating metabolic acidosis that does not add problematic counterions, such as sodium or potassium. Veverimer selectively captures and removes hydrochloric acid from the gastrointestinal tract and increases serum bicarbonate [44]. In a multicenter randomized controlled trial, treatment with veverimer improved renal outcomes, identified as occurrence of renal replacement therapy, or a decline in estimated glomerular filtration rate (eGFR) of at least $50 \%$, over 52 weeks [43]. Initial research in the veverimer trials hypothesized that chloride ions would increase with veverimer treatment. However, chloride ion levels did not increase in response to veverimer. Interestingly, administration of veverimer reduced the anion gap in CKD patients at 5, 12, and 52 weeks after trial initiation $[43,44,46]$ (Table 1). These data may suggest that veverimer improves kidney function through anion gap reduction. 
Table 1. Differences in electrolyte levels compared with baseline data in the veverimer trials

\begin{tabular}{lcccccc}
\hline Study & Year & No. of patients & $\Delta\left[\mathrm{Na}^{+}\right](\mathrm{mEq} / \mathrm{L})$ & $\Delta\left[\mathrm{Cl}^{-}\right](\mathrm{mEq} / \mathrm{L})$ & $\Delta\left[\mathrm{HCO}_{3}-\right]\left(\mathrm{mEq}^{-\mathrm{L}}\right)$ & $\Delta\left[\mathrm{AG}^{-}\right](\mathrm{mEq} / \mathrm{L})$ \\
\hline Bushinsky et al. [44] & 2018 & 104 & 0 & 0 & 3.3 & -3.3 \\
Wesson et al. [43] & 2019 & 112 & -0.4 & -0.4 & 4.7 & -4.7 \\
Wesson et al. [46] & 2019 & 124 & 0.3 & -0.2 & 4.5 & -4.0 \\
\hline
\end{tabular}

Time-dependent confounder

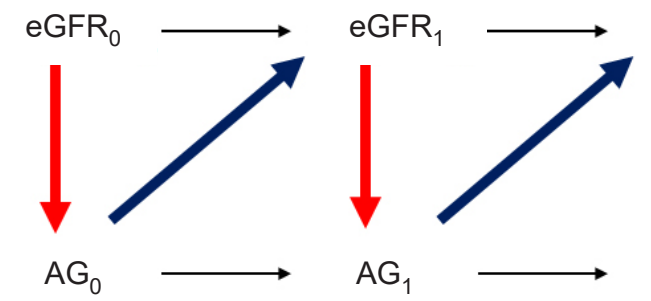

Time-dependent exposure

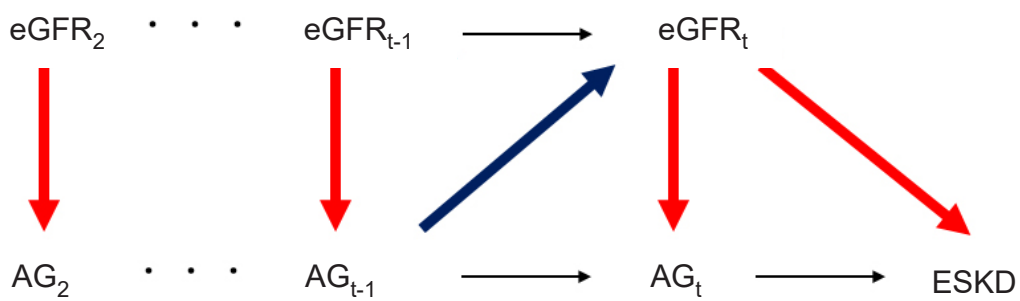

Outcome

G-methods (conterfactural model)

- Marginal structural model

- G-formula

Figure 4. Exposure-confounder feedback. Exposure-confounder feedback is generated between the anion gap (AG) and estimated glomerular filtration rate (eGFR). The eGFR can be a time-dependent confounder. Consequently, if a time-dependent confounder is identified in a statistical model, further assessment cannot be made by using conventional regression models and G-methods, like a marginal structural model or G-formula, must be adapted.

ESKD, end-stage kidney disease.

Asahina et al. [47] examined the association between anion gap and renal outcomes using cohort data from 1,168 Japanese CKD patients. It is well established that high anion gap acidosis develops during the later stages of CKD and the anion gap changes with progression of CKD. The anion gap has been repeatedly measured in conjunction with eGFR in Japanese outpatients and kidney function (eGFR) directly affects the anion gap. Moreover, an elevated anion gap may subsequently affect kidney function (eGFR). In this manner, exposure-confounder feedback is generated between the anion gap and eGFR (Fig. 4). Therefore, eGFR is believed to be a time-dependent confounder of the anion gap and renal outcomes. When the association between the anion gap and renal outcomes was analyzed in the presence of a time-dependent confounder, application of the conventional time-dependent Cox proportional hazard model alone was noted as an insufficient method for analysis as it can provide biased estimates $[48,49]$. Therefore, G-methods should be used for analysis in these situations.
G-methods include marginal structural models (MSMs) and the G-formula. MSM is explained herein for simplicity (Fig. 5); MSM is a counterfactual outcome model that is applied to account for time-dependent confounding. When applying MSMs, analysts establish imaginary pseudo-populations with or without exposure. The pseudo-populations that are established are balanced by inverse probability weighting in terms of baseline covariates and other time-dependent confounders. Asahina et al. [47] obtained time-varying inverse probability weights based on the inverse probability of treatment weights and the inverse probability of censoring weights. By analyzing differences between these pseudo-populations, they were able to determine an assumption for the exposure effect on outcomes.

As previously indicated [50], significantly elevated high anion gap acidosis occurred after stage 4 CKD [47]. In the MSM analysis, metabolic acidosis with a high anion gap was associated with a 3.04-fold rate of KFRT and a 5.56-fold rate of all-cause death, compared with the normal anion gap. 


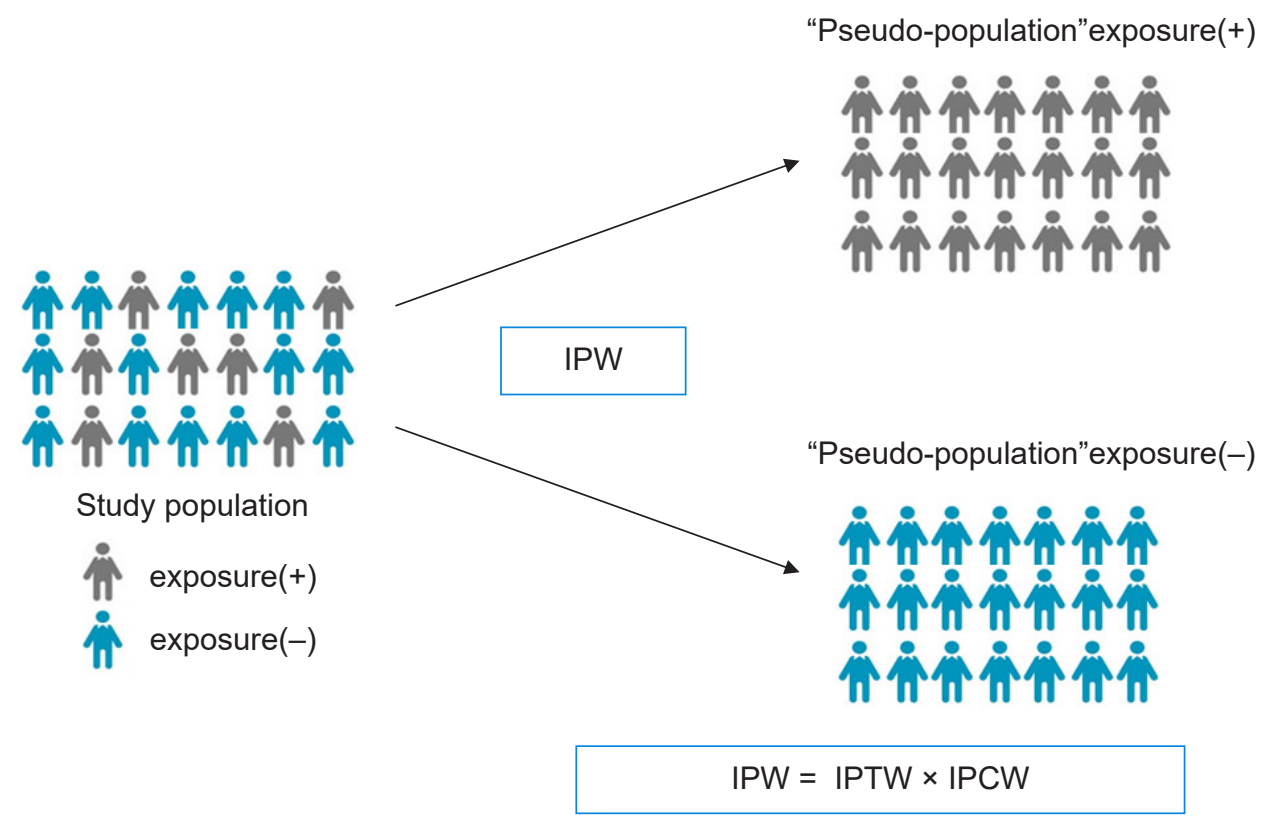

Figure 5. Marginal structural model. Kajimoto et al. [31] analyzed pseudo-populations with or without exposures. They created these pseudo-populations that were balanced by inverse probability weighting based on baseline covariates and other time-dependent confounders. By analyzing the difference between these pseudo-populations, they were able to generate assumptions for the effect of the exposure on outcomes.

IPCW, inverse probability censoring weights; IPTW, inverse probability treatment weights; IPW, inverse probability weighting.

However, in the conventional multivariate Cox proportional hazard models, high anion gap acidosis was not associated with a significantly higher rate of KFRT or all-cause death compared with normal anion gap acidosis, suggesting that analyses using Cox proportional hazard models may underestimate the association between the anion gap and renal outcome/mortality.

In a previous study of 1,145 patients with moderate CKD [51], patients in the highest traditional anion gap tertile (11.8-maximum $\mathrm{mEq} / \mathrm{L}$ ) had a higher risk of end-stage renal disease compared with adults in the middle tertile (8.1-11.8 mEq/L) using a frailty model (relative hazard, 1.76; 95\% confidence interval [CI], 1.16-2.32). The highest tertile of the full anion gap (19.54-maximum $\mathrm{mEq} / \mathrm{L}$ ) was also associated with a higher risk of all-cause mortality compared with adults in the middle tertile (15.93-19.54 mEq/ L) based on a frailty model (relative hazard, 1.20; 95\% CI, 1.01-1.39). A possible explanation for the smaller effect size in the analyses by Banerjee et al. [51], compared with those of Asahina et al. [47], may be the difference in renal function of participants (eGFR: $30-60$ vs. $10-60 \mathrm{~mL} / \mathrm{min} / 1.73 \mathrm{~m}^{2}$, respectively), rather than the difference in statistical methods.
The re-analyses that were stratified by eGFR in the study by Asahina et al. [47] revealed that high anion gap patients with an eGFR of $\geq 30 \mathrm{~mL} / \mathrm{min} / 1.73 \mathrm{~m}^{2}$ did not have a significantly higher risk of KFRT compared with normal anion gap patients within the same renal function range.

\section{Anion gap constituents in chronic kidney disease}

In the study by Asahina et al. [47], the association between a high anion gap and an increased occurrence of KFRT remained significant when the analysis was adjusted for albumin and phosphate, both of which are primary constituents of the anion gap in CKD patients, suggesting that substances other than albumin and phosphate may be involved in the progression of CKD in response to a high anion gap. In recent metabolomic analyses of human samples identified 492 uremic solutes from patients that continued on hemodialysis compared with age-matched control patients [52]. This included 214 with unknown chemical structure and 278 with known chemical structure, including wellknown uremic solutes, 3-indoxyl sulfate, p-cresol sulfate, and trimethylamine $\mathrm{N}$-oxide, all of which were reported 
to induce renal injury [39-42]. However, 3-indoxyl sulfate, p-cresol sulfate, and trimethylamine $\mathrm{N}$-oxide were only observed at low levels among the 492 uremic solutes, suggesting that other uremic solutes that comprise the anion gap in CKD patients may accelerate the progression of CKD.

Uremic solutes are effectively excreted through both glomerular filtration and tubular secretion [53,54], suggesting that tubular malfunction may lead to accumulation of uremic solutes, and accumulation of solutes may induce kidney injury. Indeed, in the Chronic Renal Insufficiency Cohort study of 3,416 CKD patients, those with reduced tubular secretion of organic acids including kynurenic acid, pyridoxic acid, indoxyl sulfate, xanthosine, isovalerylglycine, and cinnamoylglycine, were found to have a significantly higher risk of progression to CKD [55].

Recently, the human intestinal flora was reported to play a pivotal role in the production of uremic solutes [56]. Renal insufficiency itself strongly affects the colonic microenvironment and alters the composition of intestinal flora to the extent that the environment easily produces toxic uremic retention solutes $[57,58]$. Several small interventional studies targeting the intestinal flora in patients with renal insufficiency have been performed in response to these data on the association between renal function loss and changes in intestinal flora (known as dysbiosis). Such interventions, including probiotics, prebiotics, and synbiotics, were reported to decrease indoxyl sulfate or p-cresol sulfate in predialysis and dialysis patients [59-64]. However, it is largely unknown if such interventions can in fact reduce anion gap levels in patients. Therefore, further studies to elucidate the mechanism and clinical implications will be needed.

\section{Conclusions}

Assessment of venous $\mathrm{pH}$ may lead to a reduction in alkali therapy targets for CKD patients with metabolic acidosis. Therefore, it may be possible to reduce the number of cases with adverse effects from sodium bicarbonate supplementation.

Compared with sodium bicarbonate, anion gap-reducing reagents such as veverimer are a novel therapeutic approach for metabolic acidosis and may improve renal outcomes in CKD patients.

\section{Conflicts of interest}

All authors have no conflicts of interest to declare.

\section{Acknowledgments}

We thank Richard Robins, PhD, from Edanz (https:// jp.edanz.com/ac) for editing a draft of this manuscript.

\section{Authors' contributions}

Conceptualization: JYK, YS, YI

Data curation: SK, YA, TO, KH, YD

Formal analysis: YS, SK, YA

Project administration: YS

Writing-original draft: JYK

Writing-review \& editing: JYKAll authors read and approved the final manuscript.

\section{ORCID}

Jun-Ya Kaimori, https://orcid.org/0000-0001-6892-3942 Yusuke Sakaguchi, https://orcid.org/0000-0002-0987-5423 Sachio Kajimoto, https://orcid.org/0000-0001-7432-8090 Yuta Asahina, https://orcid.org/0000-0003-3640-1749 Tatsufumi Oka, https://orcid.org/0000-0002-9507-3506 Koki Hattori, https://orcid.org/0000-0002-7018-0049 Yohei Doi, https://orcid.org/0000-0002-4037-7510 Yoshitaka Isaka, https://orcid.org/0000-0002-0820-7167

\section{References}

1. Moranne O, Froissart M, Rossert J, et al. Timing of onset of CKD-related metabolic complications. J Am Soc Nephrol 2009; 20:164-171.

2. Bushinsky DA, Chabala JM, Gavrilov KL, Levi-Setti R. Effects of in vivo metabolic acidosis on midcortical bone ion composition. Am J Physiol 1999;277:F813-F819.

3. Bellasi A, Di Micco L, Santoro D, et al. Correction of metabolic acidosis improves insulin resistance in chronic kidney disease. BMC Nephrol 2016;17:158.

4. Bailey JL, Wang X, England BK, Price SR, Ding X, Mitch WE. The acidosis of chronic renal failure activates muscle proteolysis in rats by augmenting transcription of genes encoding proteins of the ATP-dependent ubiquitin-proteasome pathway. J Clin Invest 
1996;97:1447-1453.

5. Yenchek R, Ix JH, Rifkin DE, et al. Association of serum bicarbonate with incident functional limitation in older adults. Clin J Am Soc Nephrol 2014;9:2111-2116.

6. Dobre M, Gaussoin SA, Bates JT, et al. Serum bicarbonate concentration and cognitive function in hypertensive adults. Clin J Am Soc Nephrol 2018;13:596-603.

7. Kovesdy CP, Anderson JE, Kalantar-Zadeh K. Association of serum bicarbonate levels with mortality in patients with non-dialysis-dependent CKD. Nephrol Dial Transplant 2009;24:12321237.

8. Navaneethan SD, Schold JD, Arrigain S, et al. Serum bicarbonate and mortality in stage 3 and stage 4 chronic kidney disease. Clin J Am Soc Nephrol 2011;6:2395-2402.

9. Djamali A, Singh T, Melamed ML, et al. metabolic acidosis 1 year following kidney transplantation and subsequent cardiovascular events and mortality: an observational cohort study. Am J Kidney Dis 2019;73:476-485.

10. Wesson DE, Pruszynski J, Cai W, Simoni J. Acid retention with reduced glomerular filtration rate increases urine biomarkers of kidney and bone injury. Kidney Int 2017;91:914-927.

11. Nath KA, Hostetter MK, Hostetter TH. Pathophysiology of chronic tubulo-interstitial disease in rats. Interactions of dietary acid load, ammonia, and complement component C3. J Clin Invest 1985;76:667-675.

12. Phisitkul S, Hacker C, Simoni J, Tran RM, Wesson DE. Dietary protein causes a decline in the glomerular filtration rate of the remnant kidney mediated by metabolic acidosis and endothelin receptors. Kidney Int 2008;73:192-199.

13. Wesson DE, Jo CH, Simoni J. Angiotensin II-mediated GFR decline in subtotal nephrectomy is due to acid retention associated with reduced GFR. Nephrol Dial Transplant 2015;30:762-770.

14. Harambat J, Kunzmann K, Azukaitis K, et al. Metabolic acidosis is common and associates with disease progression in children with chronic kidney disease. Kidney Int 2017;92:1507-1514.

15. Dobre M, Yang W, Chen J, et al. Association of serum bicarbonate with risk of renal and cardiovascular outcomes in CKD: a report from the Chronic Renal Insufficiency Cohort (CRIC) study. Am J Kidney Dis 2013;62:670-678.

16. Raphael KL, Wei G, Baird BC, Greene T, Beddhu S. Higher serum bicarbonate levels within the normal range are associated with better survival and renal outcomes in African Americans. Kidney Int 2011;79:356-362.

17. Menon V, Tighiouart H, Vaughn NS, et al. Serum bicarbonate and long-term outcomes in CKD. Am J Kidney Dis 2010;56:907-914.
18. Shah SN, Abramowitz M, Hostetter TH, Melamed ML. Serum bicarbonate levels and the progression of kidney disease: a cohort study. Am J Kidney Dis 2009;54:270-277.

19. de Brito-Ashurst I, Varagunam M, Raftery MJ, Yaqoob MM. Bicarbonate supplementation slows progression of CKD and improves nutritional status. J Am Soc Nephrol 2009;20:2075-2084.

20. Mahajan A, Simoni J, Sheather SJ, Broglio KR, Rajab MH, Wesson DE. Daily oral sodium bicarbonate preserves glomerular filtration rate by slowing its decline in early hypertensive nephropathy. Kidney Int 2010;78:303-309.

21. Goraya N, Simoni J, Jo C, Wesson DE. Dietary acid reduction with fruits and vegetables or bicarbonate attenuates kidney injury in patients with a moderately reduced glomerular filtration rate due to hypertensive nephropathy. Kidney Int 2012;81:86-93.

22. Dubey AK, Sahoo J, Vairappan B, Haridasan S, Parameswaran S, Priyamvada PS. Correction of metabolic acidosis improves muscle mass and renal function in chronic kidney disease stages 3 and 4: a randomized controlled trial. Nephrol Dial Transplant 2020;35:121-129.

23. Di Iorio BR, Bellasi A, Raphael KL, et al. Treatment of metabolic acidosis with sodium bicarbonate delays progression of chronic kidney disease: the UBI Study. J Nephrol 2019;32:989-1001.

24. Navaneethan SD, Shao J, Buysse J, Bushinsky DA. Effects of treatment of metabolic acidosis in CKD: a systematic review and meta-analysis. Clin J Am Soc Nephrol 2019;14:1011-1020.

25. National Kidney Foundation. K/DOQI clinical practice guidelines for bone metabolism and disease in chronic kidney disease. Am J Kidney Dis 2003;42(4 Suppl 3):S1-S201.

26. Kidney Disease: Improving Global Outcomes (KDIGO) CKD Work Group. KDIGO 2012 clinical practice guideline for the evaluation and management of chronic kidney disease. Kidney Int Suppl 2013;3:1-150.

27. Rose BD, Post TW, Stokes J. Clinical physiology of acid base and electrolyte disorders. 6th ed. New York: McGraw-Hill; 2017.

28. Halperin ML, Goldstein MB. Fluid, electrolyte, and acid-base physiology. 5th ed. Philadelphia: WB Saunders; 2016.

29. Sakurabayashi I. Blood pH. In: Sakurabayashi I, Kumasaka K, Ito K, Miyaji Y, , editors. Laboratory medicine encyclopedia \& dictionary. Tokyo: Ishiyaku Publishers, Inc.; 2008.

30. Kraut JA, Raphael KL. Assessment of acid-base status: beyond serum bicarbonate. Clin J Am Soc Nephrol 2021;16:1429-1431.

31. Kajimoto S, Sakaguchi Y, Asahina Y, Kaimori JY, Isaka Y. Modulation of the association of hypobicarbonatemia and incident kidney failure with replacement therapy by venous $\mathrm{pH}$ : a cohort study. Am J Kidney Dis 2021;77:35-43. 
32. Raphael KL, Murphy RA, Shlipak MG, et al. Bicarbonate concentration, acid-base status, and mortality in the health, aging, and body composition study. Clin J Am Soc Nephrol 2016;11:308316.

33. Husted FC, Nolph KD, Maher JF. NaHCO3 and NaC1 tolerance in chronic renal failure. J Clin Invest 1975;56:414-419.

34. Husted FC, Nolph KD. NaHCO3 and $\mathrm{NaCl}$ tolerance in chronic renal failure II. Clin Nephrol 1977;7:21-25.

35. Garofalo C, Provenzano M, Andreucci M, et al. Predictive effect of salt intake on patient and kidney survival in non-dialysis CKD: competing risk analysis in older versus younger patients under nephrology care. Nephrol Dial Transplant 2021;36:22322240.

36. Kendrick J, Shah P, Andrews E, et al. Effect of treatment of metabolic acidosis on vascular endothelial function in patients with CKD: a pilot randomized cross-over study. Clin J Am Soc Nephrol 2018;13:1463-1470.

37. Madias NE. Metabolic acidosis and CKD progression. Clin J Am Soc Nephrol 2021;16:310-312.

38. Kim HJ, Kang E, Ryu H, et al. Metabolic acidosis is associated with pulse wave velocity in chronic kidney disease: results from the KNOW-CKD Study. Sci Rep 2019;9:16139.

39. Duranton F, Cohen G, De Smet R, et al. Normal and pathologic concentrations of uremic toxins. J Am Soc Nephrol 2012;23: 1258-1270.

40. Wang X, Yang S, Li S, et al. Aberrant gut microbiota alters host metabolome and impacts renal failure in humans and rodents. Gut 2020;69:2131-2142.

41. Vanholder R, Schepers E, Pletinck A, Nagler EV, Glorieux G. The uremic toxicity of indoxyl sulfate and p-cresyl sulfate: a systematic review. J Am Soc Nephrol 2014;25:1897-1907.

42. Gupta N, Buffa JA, Roberts AB, et al. Targeted inhibition of gut microbial trimethylamine $\mathrm{N}$-oxide production reduces renal tubulointerstitial fibrosis and functional impairment in a murine model of chronic kidney disease. Arterioscler Thromb Vasc Biol 2020;40:1239-1255.

43. Wesson DE, Mathur V, Tangri N, et al. Long-term safety and efficacy of veverimer in patients with metabolic acidosis in chronic kidney disease: a multicentre, randomised, blinded, placebo-controlled, 40-week extension. Lancet 2019;394:396-406.

44. Bushinsky DA, Hostetter T, Klaerner G, et al. Randomized, controlled trial of TRC101 to increase serum bicarbonate in patients with CKD. Clin J Am Soc Nephrol 2018;13:26-35.

45. Adrogué HJ, Madias NE. Veverimer: an emerging potential treatment option for managing the metabolic acidosis of CKD. Am J
Kidney Dis 2020;76:861-867.

46. Wesson DE, Mathur V, Tangri N, et al. Veverimer versus placebo in patients with metabolic acidosis associated with chronic kidney disease: a multicentre, randomised, double-blind, controlled, phase 3 trial. Lancet 2019;393:1417-1427.

47. Asahina Y, Sakaguchi Y, Kajimoto S, et al. Association of time-updated anion gap with risk of kidney failure in advanced CKD: a cohort study. Am J Kidney Dis 2022;79:374-382.

48. Mansournia MA, Etminan M, Danaei G, Kaufman JS, Collins G. Handling time varying confounding in observational research. BMJ 2017;359:j4587.

49. Naimi AI, Cole SR, Kennedy EH. An introduction to g methods. Int J Epidemiol 2017;46:756-762.

50. Tanemoto M. Gap acidosis except lactic acidosis develops and progresses during chronic kidney disease stage G5. Clin Exp Nephrol 2019;23:1045-1049.

51. Banerjee T, Crews DC, Wesson DE, et al. Elevated serum anion gap in adults with moderate chronic kidney disease increases risk for progression to end-stage renal disease. Am J Physiol Renal Physiol 2019;316:F1244-F1253.

52. Mair RD, Sirich TL, Plummer NS, Meyer TW. Characteristics of colon-derived uremic solutes. Clin J Am Soc Nephrol 2018;13: 1398-1404.

53. Koepsell H, Endou H. The SLC22 drug transporter family. Pflugers Arch 2004;447:666-676.

54. Nigam SK. What do drug transporters really do? Nat Rev Drug Discov 2015;14:29-44.

55. Chen Y, Zelnick LR, Hoofnagle AN, et al. Prediction of kidney drug clearance: a comparison of tubular secretory clearance and glomerular filtration rate. J Am Soc Nephrol 2021;32:459-468.

56. Aronov PA, Luo FJ, Plummer NS, et al. Colonic contribution to uremic solutes. J Am Soc Nephrol 2011;22:1769-1776.

57. Poesen R, Windey K, Neven E, et al. The influence of CKD on colonic microbial metabolism. J Am Soc Nephrol 2016;27:13891399.

58. Chen Y, Zelnick LR, Wang K, et al. Kidney clearance of secretory solutes is associated with progression of CKD: the CRIC study. $J$ Am Soc Nephrol 2020;31:817-827.

59. Takayama F, Taki K, Niwa T. Bifidobacterium in gastro-resistant seamless capsule reduces serum levels of indoxyl sulfate in patients on hemodialysis. Am J Kidney Dis 2003;41(3 Suppl 1):S142-S145.

60. Meijers BK, De Preter V, Verbeke K, Vanrenterghem Y, Evenepoel P. p-Cresyl sulfate serum concentrations in haemodialysis patients are reduced by the prebiotic oligofructose-enriched 
inulin. Nephrol Dial Transplant 2010;25:219-224.

61. Sirich TL, Plummer NS, Gardner CD, Hostetter TH, Meyer TW. Effect of increasing dietary fiber on plasma levels of colon-derived solutes in hemodialysis patients. Clin J Am Soc Nephrol 2014;9:1603-1610.

62. Nakabayashi I, Nakamura M, Kawakami K, et al. Effects of synbiotic treatment on serum level of p-cresol in haemodialysis patients: a preliminary study. Nephrol Dial Transplant 2011;26:1094-1098.
63. Guida B, Germanò R, Trio R, et al. Effect of short-term synbiotic treatment on plasma p-cresol levels in patients with chronic renal failure: a randomized clinical trial. Nutr Metab Cardiovasc Dis 2014;24:1043-1049.

64. Rossi M, Johnson DW, Morrison M, et al. Synbiotics easing renal failure by improving gut microbiology (SYNERGY): a randomized trial. Clin J Am Soc Nephrol 2016;11:223-231. 